\title{
True Atomic-Resolution Imaging of (1014) Calcite in Aqueous Solution by Frequency Modulation Atomic Force Microscopy
}

\author{
Sebastian Rode, ${ }^{\dagger}$ Noriaki Oyabu, ${ }^{\ddagger}$ Kei Kobayashi, ${ }^{\ddagger}$ Hirofumi Yamada ${ }^{\ddagger}$ and \\ Angelika Kühnle*,† \\ Fachbereich Physik, Universität Osnabrück, Barbarastr. 7, 49076 Osnabrück, Germany, and Department
of Electronic Science and Engineering, Kyoto University, Katsura, Nishikyo, Kyoto 615-8510, Japan
}

Received October 16, 2008. Revised Manuscript Received January 2, 2009

\begin{abstract}
Calcite $\left(\mathrm{CaCO}_{3}\right)$ is one of the most abundant minerals on earth and plays an important role in a wide range of different fields including, for example, biomineralization and environmental geochemistry. Consequently, surface processes and reactions such as dissolution and growth as well as (macro)molecule adsorption are of greatest interest for both applied as well as fundamental research. An in-depth understanding of these processes requires knowledge about the detailed surface structure in its natural state which is quite often a liquid environment. We have studied the most stable cleavage plane of calcite under liquid conditions using frequency modulation atomic force microscopy. Using this technique, we achieved true atomic-resolution imaging, demonstrating the high-resolution capability of frequency modulation atomic force microscopy in liquids. We could reproduce contrast features reported before using contact mode atomic force microscopy, originating from the protruding oxygen atom of the carbonate groups. Besides this contrast, however, our results, indeed, indicate that we obtain more detailed structural information, revealing the chilcium sublattice of the $(10 \overline{1} 4)$ cleavage plane.
\end{abstract}

\section{Introduction}

Calcite, the most stable polymorph of $\mathrm{CaCO}_{3}$, is abundant in the geological environment and an extremely important mineral in a wide range of different fields. Calcite plays a crucial role in biomineralization, where crystal growth is controlled by the presence of biological macromolecules. ${ }^{1} \mathrm{CaCO}_{3}$ precipitates upon scaling, a process that is undesirable in many industrial as well as daily applications such as water desalination ${ }^{2}$ and laundry. ${ }^{3}$ Calcite is used in a number of industrial products, for example, cement, paint, cosmetics, paper, and pharmaceuticals, and, due to its birefringence, in optical devices. Moreover, calcite has been discussed in the context of homochirality of life, as enantiospecific adsorption of natural amino acids to calcite surfaces has been demonstrated. ${ }^{4-6}$

Calcite has a rhombohedral crystal structure and belongs to the $R \overline{3} c$ space group. The calcite unit cell is shown in Figure 1a. The vectors $\mathbf{a}$ and $\mathbf{b}$ are of same length $(a=b=0.499 \mathrm{~nm})$ and include an angle $\gamma$ of $120^{\circ}$, and the vector $\mathbf{c}$ is perpendicular to the plane spanned by $\mathbf{a}$ and $\mathbf{b}(c=1.706 \mathrm{~nm})$. The most stable cleavage plane is the (1014) plane, indicated by dashed lines in Figure 1a. Within this plane, both calcium and carbonate ions form rectangular sublattices, as shown in the truncated bulk structure in Figure 1b. The surface unit cell has dimensions of $a_{1}=0.499 \mathrm{~nm}$ and $a_{2}=0.810 \mathrm{~nm}$. It consists of two calcium ions and two carbonate groups $\left(\mathrm{CO}_{3}{ }^{2-}\right)$. The carbonate groups are planar with one oxygen atom within the (1014) cleavage plane, one above and one below, indicated by the size of the circles representing oxygen atoms. When considering the

* To whom correspondence should be addressed. E-mail: kuehnle@ uos.de.

${ }^{\dagger}$ Universität Osnabrück.

* Kyoto University.

(1) Addadi, L.; Weiner, S. Angew. Chem., Int. Ed. Engl. 1992, 31(2), 153-169.

(2) Rieger, J.; Thieme, J.; Schmidt, C. Langmuir 2000, 16(22), 8300-8305.

(3) Hadicke, E.; Rieger, J.; Rau, I. U.; Boeckh, D. Phys. Chem. Chem. Phys. 1999, 1(17), 3891-3898.

(4) Addadi, L.; Weiner, S. Nature 2001, 411, 753-755.

(5) Hazen, R. M.; Filley, T. R.; Goodfriend, G. A. Proc. Nat. Acad. Sci. U.S.A. 2001, 98(10), 5487-5490.

(6) Orme, C. A.; Noy, A.; Wierzbicki, A.; McBride, M. T.; Grantham, M.; Teng, H. H.; Dove, P. M.; DeYoreo, J. J. Nature 2001, 411, 775-779.
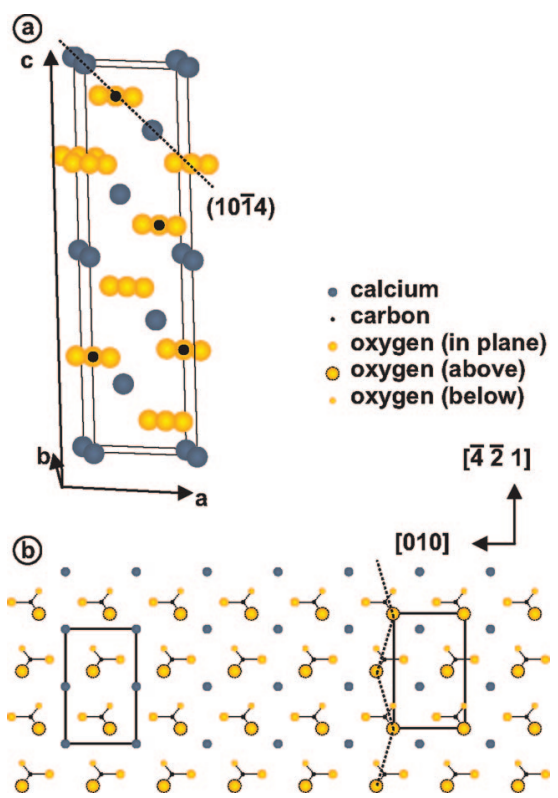

Figure 1. Calcite unit cell and cleavage plane. (a) Hexagonal calcite unit cell. Calcium, carbon, and oxygen atoms are represented by blue, black, and yellow balls, respectively. The (1014) cleavage plane is indicated by a dashed line. (b) Truncated bulk surface structure of the (1014) cleavage plane. Two different unit cells are indicated, considering the calcium ions (left) or the protruding oxygen atoms (right).

protruding oxygen atoms of the carbonate groups, a zigzag structure is formed along the $[\overline{4} \overline{2} 1]$ direction, as shown to the right in Figure 1b. The two carbonate groups within the unit cell are inequivalent as the in-plane oxygen atoms point in opposite directions.

The surface structure ${ }^{7-13}$ as well as surface reactions such as dissolution and growth ${ }^{14,15}$ of the (1014) calcite surface have been studied in great detail both under ultrahigh vacuum conditions and in ambient or liquid environment. Using atomic force microscopy (AFM) operated in the contact mode, several structural details of the (1014) cleavage plane have been revealed, 
including the lattice dimensions of the surface unit cell. ${ }^{10,11}$ Liang et al. have presented contact mode AFM images that exhibit the characteristic zigzag structure of the protruding oxygen atoms. Slight differences in the atomic corrugation have been interpreted as relaxations of the carbonate groups due to rotation or distortion. ${ }^{13}$ These relaxations result in an apparent "pairing" of the protruding oxygen atoms that have been reported before by Stipp et al. ${ }^{12}$ Besides the pairing, Stipp et al. report on height variations along the $\left[\begin{array}{lll}0 & 1 & 0\end{array}\right]$ direction, resulting in a $(2 \times 1)$ structure. This $(2 \times 1)$ structure has also been found in lowenergy electron diffraction (LEED) ${ }^{7}$ and modeling studies. ${ }^{16,17}$ Other authors report on contact mode AFM results revealing a unit cell with dimensions of $a_{1}=0.5 \mathrm{~nm}$ and $a_{2}^{\prime}=0.4 \mathrm{~nm}$, corresponding to the calcium sublattice. ${ }^{8}$ These differences in appearance using contact mode AFM strongly suggests that imaging conditions can have a crucial influence on contrast formation. Moreover, contact mode AFM usually lacks atomic resolution, as resolution is limited to the size of the tip. Operated under liquid conditions, it has been argued that water molecules may act as a lubricant layer, enabling true atomic resolution imaging. ${ }^{10,13}$ The influence of tip size and forces acting onto the surface when performing contact mode AFM images does, however, remain. This is true for all AFM modes; however, recent high-resolution data suggest that a precise control of the tip-sample interaction can be realized by frequency modulation AFM (FM-AFM). This technique has proven to constitute a promising method of atomic-resolution imaging in liquids. ${ }^{18,19}$

In this work, we present the first FM-AFM measurements of the calcite $(10 \overline{1} 4)$ cleavage plane under liquid conditions. We have obtained true atomic resolution images, exhibiting different contrasts. We could reproduce both the zigzag pattern and the row pairing known to originate from the protruding oxygen rows as well as the rectangular structure with reduced unit cell dimensions ascribed to the calcium sublattice. Besides these contrasts, our results reveal further structural details that can be ascribed to simultaneous imaging of both carbonate groups and calcium ions, demonstrating the high-resolution capabilities of frequency modulation AFM in liquids.

\section{Experimental Procedure}

Experiments were performed in liquids using a newly developed Shimadzu atomic force microscope with a home-built controller and an open fluid cell. As force sensors, we used gold-coated, p-doped silicon cantilevers (PPP-NCHAuD from Nanosensors, Neuchâtel, Switzerland) with an eigenfrequency of about $160 \mathrm{kHz}$, a spring constant of about $40 \mathrm{~N} / \mathrm{m}$, and a $Q$ value around 8 in Milli-Q water.

(7) Stipp, S. L.; Hochella, M. F. Geochim. Cosmochim. Acta 1991, 55(6), 1723-1736.

(8) Hillner, P. E.; Manne, S.; Gratz, A. J.; Hansma, P. K. Ultramicroscopy 1992, 42, 1387-1393.

(9) Rachlin, A. L.; Henderson, G. S.; Goh, M. C. Am. Mineral. 1992, 77(9-10), 904-910.

(10) Ohnesorge, F.; Binnig, G. Science 1993, 260(5113), 1451-1456.

(11) Raina, G.; Gauldie, R. W.; Sharma, S. K.; Helsley, C. E. Ferroelectr. Lett. Sect. 1994, 17(3-4), 65-72.

(12) Stipp, S. L. S.; Eggleston, C. M.; Nielsen, B. S. Geochim. Cosmochim. Acta 1994, 58(14), 3023-3033.

(13) Liang, Y.; Lea, A. S.; Baer, D. R.; Engelhard, M. H. Surf. Sci. 1996, 351(1-3), 172-182.

(18) Fukuma, T.; Kobayashi, K.; Matsushige, K.; Yamada, H. Appl. Phys. Lett. 2005, 87(3), 034101.

(19) Hogan, J. Nature 2006, 440(7080), 14-15.

(14) Dove, P. M.; Hochella, M. F. Geochim. Cosmochim. Acta 1993, 57(3), $705-714$.

(15) Gratz, A. J.; Hillner, P. E.; Hansma, P. K. Geochim. Cosmochim. Acta 1993, 57(2), 491-495.

(16) Kristensen, R.; Stipp, S. L. S.; Refson, K. J. Chem. Phys. 2004, 121(17), $8511-8523$. 925.
The cantilever oscillation amplitude was kept constant at a level of $0.2-2.0 \mathrm{~nm}$; images shown here were taken with amplitudes in the range of $0.2-0.5 \mathrm{~nm}$. All images were taken in frequency modulation mode as described before. ${ }^{18}$ Best images were obtained in constant height mode, allowing for fast scan speeds up to 0.4 frames/s. When scanning in constant height mode, the information is recorded in the frequency shift (df), resulting in a df contrast image. For the amplitudes used here, a positive frequency shift setpoint corresponds to imaging in the repulsive regime. ${ }^{20}$

Calcite samples were of optical quality from Korth Kristalle $\mathrm{GmbH}$ (Kiel, Germany). The calcite samples were cleaved immediately before inserting them into the fluid cell and placing a solution droplet onto the surface. We cleaved the samples by gently scoring a line parallel to the edge of the crystal with a scalpel, a recipe known to produce flat (1014) cleavage planes. ${ }^{7}$ Aqueous solutions were prepared using Milli-Q water. A solution droplet with a volume of $100 \mu \mathrm{L}$ was placed onto the surface using a pipet. Images were taken both in pure Milli-Q water as well as in solutions of $\mathrm{KCl}$ with concentrations ranging from 0.15 to $1 \mathrm{M}$. Imaging seemed to be facilitated in the presence of $\mathrm{KCl}$. We could, however, not observe a reliable dependence of the imaging contrast on the ionic strength. Since we used an open fluid cell, the droplet volume was not constant during the measurements but subjected to evaporation. Before scanning, the cantilever was immersed into the liquid and the entire setup was allowed to equilibrate, a procedure that was found to be important for obtaining atomically resolved images. ${ }^{9}$

We have carefully evaluated the drift in all images. Drift in the $x y$-plane was observed to have a significant influence on images taken with slow scan speed, while fast scanning as well as temperature control and increased humidity resulted in nearly drift-free images. We used a commercial incubator (Cool incubator $\mathrm{CN}-40 \mathrm{~A}$ from Mitsubishi Electric Engineering Co., Ltd.), allowing for stabilizing the temperature at $298 \pm 0.15 \mathrm{~K}$ for $10 \mathrm{~h}$. Moreover, we reduced the rate of droplet evaporation by placing Petri dishes filled with Milli-Q water into the incubator, thereby increasing the humidity. Images showing a clear defect site serving as a landmark (such as, e.g., Figure 2a) can be easily drift-corrected. Performing a drift correction for Figure $2 \mathrm{a}$ results in an image that does, indeed, not differ from the original image, demonstrating the fact that drift is negligible in this image. Similarly, we have confirmed that drift is negligible in all images shown here by comparing consecutive images of opposite slow scan direction (up and down scans). All images presented are raw data except for a plane subtraction.

The images shown here are a selection from roughly 3000 highquality images with atomic or near-atomic resolution taken during 12 independent sessions. For all sessions, we started with a freshly cleaved calcite sample and a new cantilever. Additionally, cantilevers were exchanged during a session if the image quality degraded. The selected images represent typical examples of high-quality images, thus providing a representative picture of the observed contrast modes.

\section{Results and Discussion}

Depending on imaging conditions such as scanning speed, scanning angle, and tip condition, images were achieved differing slightly in appearance as well as images exhibiting a qualitatively different contrast. True atomic resolution was obtained, demonstrated by imaging step edges of surface defects. An image exhibiting a surface defect is shown in Figure 2a, revealing a bright atomic-scale defect (arrow). An atomically resolved step edge is shown in Figure $2 b$.

We observe two qualitatively different contrast patterns. One imaging contrast reveals a characteristic zigzag pattern that can be unambiguously ascribed to the protruding oxygen atoms of the carbonate groups as previously reported in the literature. ${ }^{12,13}$ The df images shown in Figure 3 belong to this type of imaging contrast, which is characterized by the zigzag rows formed along

(20) Rahe, P.; Bechstein, R.; Schütte, J.; Ostendorf, F.; Kühnle, A. Phys. Rev. B 2008, 77(19), 195410. 

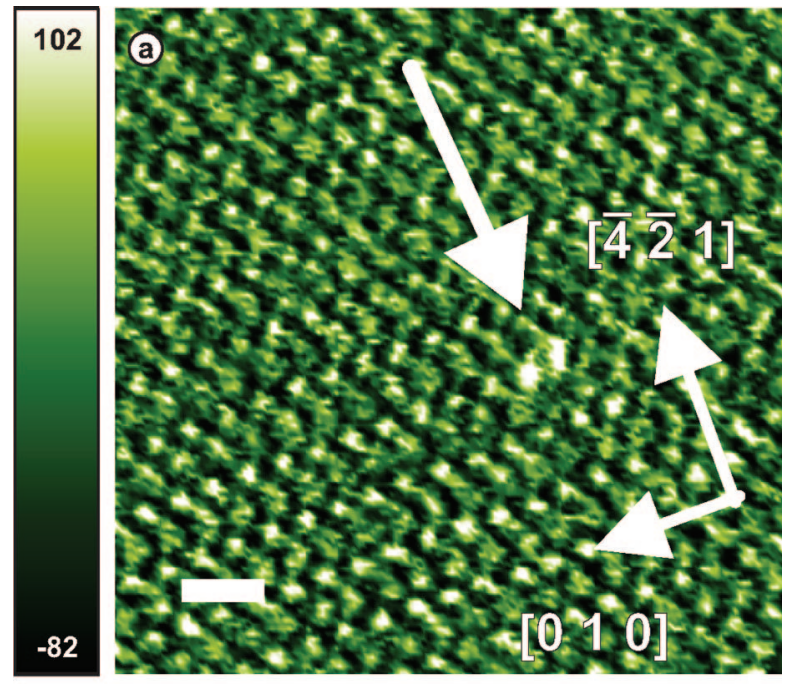

59

$-151$

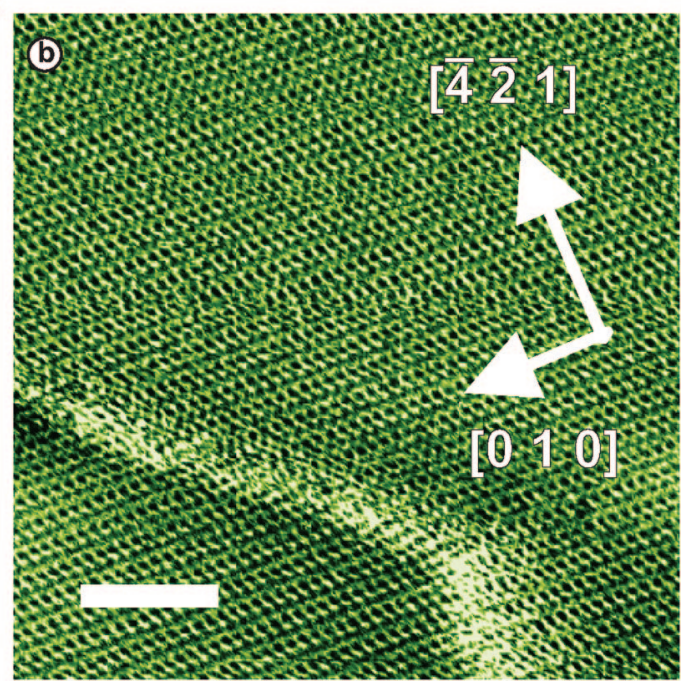

Figure 2. Atomically resolved images of the (1014) calcite cleavage plane exhibiting a surface defect and a step edge. (a) df contrast taken at a frequency shift of $+3 \mathrm{~Hz}$ and an amplitude of $0.3 \mathrm{~nm}$ in Milli-Q water. Scale bar is $1 \mathrm{~nm}$. (b) df contrast taken at a frequency shift of $+60 \mathrm{~Hz}$ and an amplitude of $0.3 \mathrm{~nm}$ in Milli-Q water. Scale bar is 4 $\mathrm{nm}$.

the $[\overline{4} \overline{2} 1]$ direction and unit cell dimensions of $a_{1}=0.5 \mathrm{~nm}$ along the [ $\left[\begin{array}{lll}0 & 1 & 0\end{array}\right]$ direction and $a_{2}=0.8 \mathrm{~nm}$ along the [ $\left.\overline{4} \overline{2} 1\right]$ direction. The characteristic zigzag line is indicated in Figure 3a. Three effects have been discussed in the context of this imaging contrast, which have been ascribed to distortions such as rotations of one carbonate group: First, the two oxygen atoms within the unit cell can appear as pair of two dots, resulting in "row pairing" as indicated by the ellipses in Figure $3 b$. Second, the two oxygen atoms within the unit cell appear in different brightness, as shown in Figure 3c, leading to rows of bright dots that are separated by rows of less bright dots. The difference in appearance can be as pronounced as in Figure 3d, eventually resulting in a rectangular structure formed by the bright protruding oxygen atoms. Third, Stipp and co-workers have reported on a $(2 \times 1)$ superstructure, caused by slight apparent height deviations of the protruding

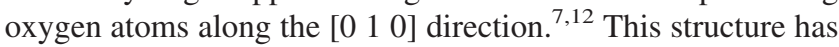
been addressed by modeling studies and was ascribed to surface phonons ${ }^{17}$ and discussed to be step-induced. ${ }^{16}$ We have no strong evidence for this $(2 \times 1)$ modulation along the $\left[\begin{array}{lll}0 & 1 & 0\end{array}\right]$ direction. Some images show slight variations in the apparent height; however, in the majority of the images, no modulations were observed.
Interestingly, in addition to this pattern produced by the protruding oxygen atoms, our images reveal further structural details that can be ascribed to calcium ions (see Figure $3 b$ ). Simultaneous imaging of both the protruding oxygen atoms and the calcium ions has been demonstrated only one time so far. This high resolution was achieved by Ohnesorge and Binnig when operating the contact mode atomic force microscope in the attractive regime. ${ }^{10}$ Two calcium ions exist within the unit cell. However, we often observe only one of the calcium ions within the unit cell as seen in Figure $3 c$. This is an effect we attribute to the imaging conditions and not to a nonstoichiometric surface, ${ }^{21}$ as we observe the images to change from showing only one calcium atom of the unit cell to showing both atoms. The calcium features in between oxygen features often result in a characteristic appearance of dots that are connected to form a row along the $\left[\begin{array}{lll}0 & 1 & 0\end{array}\right]$ direction. In Figure 3b, both calcium ions within the unit cell can be identified as less-bright features in between the oxygen atoms.

Besides imaging the protruding oxygen atoms, we occasionally observe a qualitatively different contrast as shown in Figure 4. This contrast is characterized by a rectangular unit cell with a size half as large as the (1014) unit cell, resembling the structure that has been presented by Hillner et al. ${ }^{22}$ Such a structure agrees with both the calcium and carbon sublattice, and it cannot be concluded from the basis of these AFM images solely which sublattice causes the pattern. It seems, however, more likely to image the calcium sublattice, as the carbon atoms are surrounded by oxygen atoms.

The change between the contrast pattern shown in Figures 3 and 4 is not related to changes in the ionic strength but happens occasionally. We thus suppose a tip change to be responsible for this change in contrast.

The different appearance in Figure 3 and the qualitatively different contrast (Figure 4) observed here are attributed to different scanning conditions such as scanning speed, scanning angle, as well as tip termination. Several groups discuss the issue of angle-dependent appearance of calcite in AFM measurements, and it has been suggested that the carbonate group might experience a tip-induced displacement and rotation. ${ }^{23}$ These relaxation effects might be responsible for the multitude of slightly differing appearances observed in Figure 3.

As FM-AFM offers an enhanced sensitivity compared to contact mode AFM, FM-AFM might be capable of detecting electrostatic interactions rather than pure Pauli repulsion. As we measure with amplitudes larger than $0.1 \mathrm{~nm}$, the positive frequency shift setpoints used here correspond to repulsive interactions. $^{20} \mathrm{~A}$ positive tip termination could, therefore, be sensitive to cations, similar to what is known in several examples of FM-AFM under ultrahigh vacuum conditions. ${ }^{24,25}$ The applicability of this simple picture of purely electrostatic interaction might, of course, be limited in the case of a rather complex environment such as liquids. However, this simple picture would explain why different contrasts are observed in these images. According to this picture, the imaging contrast presented in Figure 4 would originate from scanning with a

(21) Kerisit, S.; Marmier, A.; Parker, S. C. J. Phys. Chem. B 2005, 109(39), 18211-18213.

(22) Hillner, P. E.; Gratz, A. J.; Manne, S.; Hansma, P. K. Geology 1992, 20(4), 359-362.

(23) Hofer, W. A.; Foster, A. S.; Shluger, A. L. Rev. Mod. Phys. 2003, 75, 1287-1331.

(24) Foster, A. S.; Barth, C.; Shluger, A. L.; Reichling, M. Phys. Rev. Lett. 2001, 86(11), 2373-2376.

(25) Lauritsen, J. V.; Foster, A. S.; Olesen, G. H.; Christensen, M. C.; Kühnle, A.; Helveg, S.; Rostrup-Nielsen, J. R.; Clausen, B. S.; Reichling, M.; Besenbacher, F. Nanotechnology 2006, 17 (14), 3436-3441. 

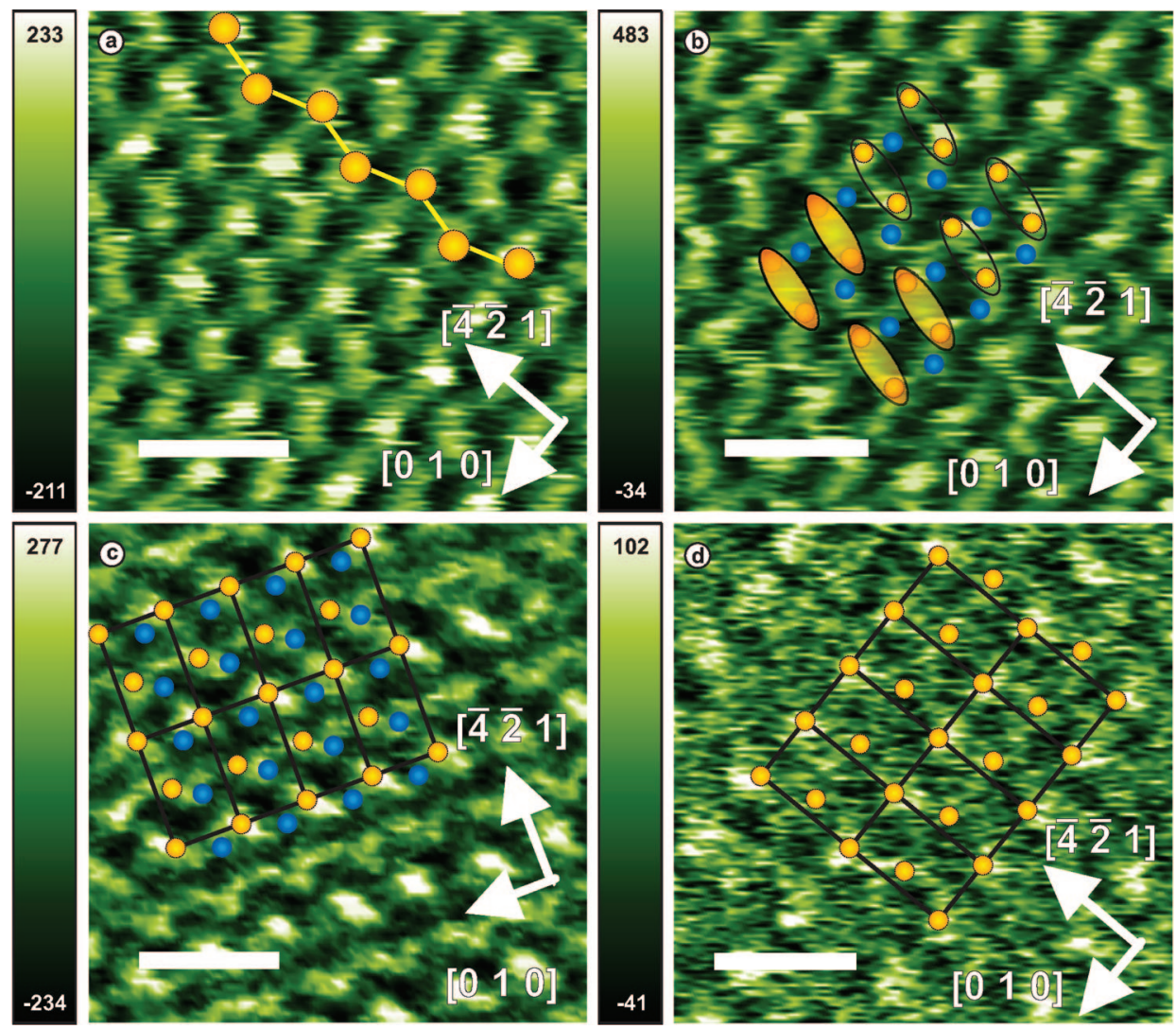

Figure 3. Atomically resolved images showing a frequently observed contrast mode that can be ascribed to the protruding oxygen atoms. Scale bars are $1 \mathrm{~nm}$. (a) df contrast image taken at a frequency shift of $+84 \mathrm{~Hz}$ and an amplitude of $0.2 \mathrm{~nm}$ in $1 \mathrm{M} \mathrm{KCl}$. (b) df contrast image taken at a frequency shift of $+82 \mathrm{~Hz}$ and an amplitude of $0.2 \mathrm{~nm}$ in $1 \mathrm{M} \mathrm{KCl}$. (c) df contrast image taken at a frequency shift of $+140 \mathrm{~Hz}$ and an amplitude of $0.3 \mathrm{~nm}$ in Milli-Q water. (d) df contrast image taken at a frequency shift of $+38 \mathrm{~Hz}$ and an amplitude of $0.2 \mathrm{~nm}$ in $1 \mathrm{M} \mathrm{KCl}$.

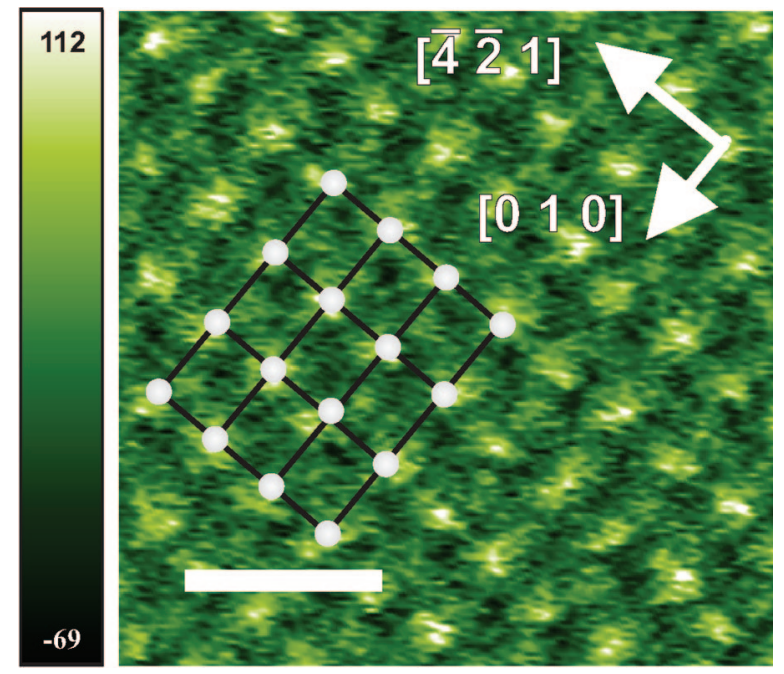

Figure 4. Atomically resolved images showing another observed contrast mode. Scale bar is $1 \mathrm{~nm}$. df contrast image taken at a frequency shift of $+23 \mathrm{~Hz}$ and an amplitude of $0.2 \mathrm{~nm}$ in $1 \mathrm{M} \mathrm{KCl}$.

positively terminated tip. A negative tip termination could, on the other hand, be responsible for imaging the protruding oxygen atoms solely.

Significant tip-induced surface relaxations, imaging of adsorbates, as well as screening effects through ions from the surrounding liquid might as well influence the contrast formation.
Theoretical input as well as further experimental work are required for developing a comprehensive model that accounts for all structural details observed here.

\section{Conclusions}

True atomic resolution was obtained on the (1014) cleavage plane of calcite using FM-AFM in liquids operated in the repulsive regime. Very small amplitudes between 0.2 and $0.5 \mathrm{~nm}$ were used, enhancing the sensitivity by limiting the oscillation to shortrange forces. We could reproduce contact mode AFM contrasts reported upon in the literature. Compared to previous contact mode AFM images, however, our results reveal further structural details that are ascribed to simultaneously imaging the calcium and carbonate sublattice. The increased resolution is attributed to the increased sensitivity toward small, yet structurally decisive changes in the tip-sample interaction. Our study thus demonstrates the high-resolution capabilities of FM-AFM for operation in liquid environments.

Acknowledgment. This work was supported by the Japan Science and Technology Agency (JST) program "Development of System and Technology for Advanced measurement and Analysis" through the project "Developments of High-Resolution Analytical Force Microscopy Working in Various Environments". Financial support from the Deutsche Forschungsgemeinschaft (DFG) through an Emmy Noether grant (A.K.) and the GRK695 (S.R.) is gratefully acknowledged.

\section{LA803448V}

